\title{
Case Report: Teriparatide treatment in a case of severe pregnancy -and lactation- associated osteoporosis
}

\author{
Kalliopi Lampropoulou-Adamidou, George Trovas, Ioannis P. Stathopoulos, \\ Nikolaos A. Papaioannou
}

Laboratory for the Research of the Musculoskeletal System “Th. Garofalidis”, KAT Hospital, Athens, Greece

\begin{abstract}
OBJECTIVE: Pregnancy- and lactation-associated osteoporosis (PLO) is an uncommon disease. The majority of cases are seen in the third trimester or early post-partum in primagravid women and the prominent clinical feature of PLO is severe and prolonged back pain and height loss. The prevalence and aetiology of this disorder are as yet unclear and there are no guidelines for its treatment. CASE REPORT: We report the outcomes of teriparatide (TRP) treatment in a woman suffering from severe PLO with 6 vertebral fragility fractures, severe back pain and very low BMD. RESULTS: Thirteen months after the initiation of therapy, the patient was almost free of back pain. There was no new clinical vertebral fracture. Her laboratory tests were all normal. BMD increased by $24.4 \%$ at the lumbar spine, $9.9 \%$ and $4.6 \%$ at the left and the right total hip and $\mathbf{1 2 . 6 \%}$ and $\mathbf{7 . 8 \%}$ at the left and right femur neck, respectively. CONCLUSION: TRP treatment simultaneously with weaning and calcium and vitamin $D$ supplementation seems to considerably increase BMD, improve severe back pain and quality of life and prevent further occurrence of vertebral fractures, making TRP a helpful tool in restoring bone strength in PLO patients.
\end{abstract}

Key words: Lactation, Osteoporosis, Pregnancy, Premenopausal osteoporosis, Teriparatide

\section{INTRODUCTION}

Pregnancy- and lactation-associated osteoporosis (PLO) is an uncommon disease. The first description

Address for correspondence:

Kalliopi Lampropoulou-Adamidou, MD, MSc, Laboratory for the Research of the Musculoskeletal System

"Th. Garofalidis", KAT General Hospital of Athens, 10

Athinas Str., 14561, Kifissia, Athens, Greece,

Tel.: +306984229202, +302108018123, Fax: +302108018122,

E-mail: kilampropoulou@gmail.com

Received 22-04-12, Revised 15-07-12, Accepted 24-08-12 of PLO was made by Nordin et al in $1955 .{ }^{1}$ It is characterised by the occurrence of fragility fracture(s), most commonly involving the lower thoracic and/ or lumbar vertebral bodies. The majority of cases are seen in the third trimester or early post-partum in primagravid women and the prominent clinical feature of PLO is severe and prolonged back pain and height loss. The prevalence and the ae tiology of this disorder are unclear. To date, more than 120 cases have been reported, mostly as individual case reports. ${ }^{2}$ Due to the lack of controlled trials and the rarity of the disease there are no formal guidelines. 
Many patients have been treated with weaning and calcium supplementation, while some of them have received additional vitamin D. ${ }^{3}$ Bisphosphonates (BPs) are used increasingly in patients suffering from PLO, resulting in a better outcome compared to that of a plain supplementation with calcium and vitamin D. However, the safety of their use by women of reproductive age has not as yet been established. ${ }^{4}$ Therefore, alternative treatment of PLO, such as teriparatide (TRP), must be considered. Here we report the outcomes of TRP treatment in a woman suffering from severe PLO with 6 vertebral fragility fractures, severe back pain and very low BMD.

\section{CASE REPORT}

A lactating 40-year-old Caucasian woman had back pain without trauma a few days after delivery of her first baby. She was treated elsewhere with physiotherapies and nonsteroidal anti-inflammatory drugs (NSAIDs). Her pain continued to worsen and 40 days after delivery she could not get up from bed nor hold her baby, although she was receiving about $75 \mathrm{mg}$ of diclofenac six times daily. MRI examination of the thoracic and lumbar spine recommended by another physician revealed 3 vertebral fractures at $\mathrm{T} 7, \mathrm{~T} 9$ and T10. One month later, she underwent a second MRI examination that detected 3 new vertebral fractures at T8, T12 and L1 (Figure). She was then referred to our centre.

Her medical history revealed that she had had thyroid nodules treated 10 years previously with levothyroxine $100 \mu \mathrm{g} /$ day for one year. She had menarche at 12 years of age and her menstrual cycle was regular. She had never smoked or consumed alcohol. She suffered a radius fracture in childhood. Her mother had osteoporosis without fractures. Her height was $158 \mathrm{~cm}$ and her weight was $56 \mathrm{~kg}$ (body mass index $22.4 \mathrm{~kg} / \mathrm{m}^{2}$ ). She reported that her previous height was $162 \mathrm{~cm}$, demonstrating a $4 \mathrm{~cm}$ height loss.

She took oral contraceptive pills for 21 days and through in vitro fertilisation she had successfully conceived. Also, she received low-molecular-weight heparin (LMWH) for thrombofilia in the last 2 trimesters of pregnancy. During pregnancy, she received 500 mg daily calcium supplementation and her nutritional calcium intake was satisfactory.

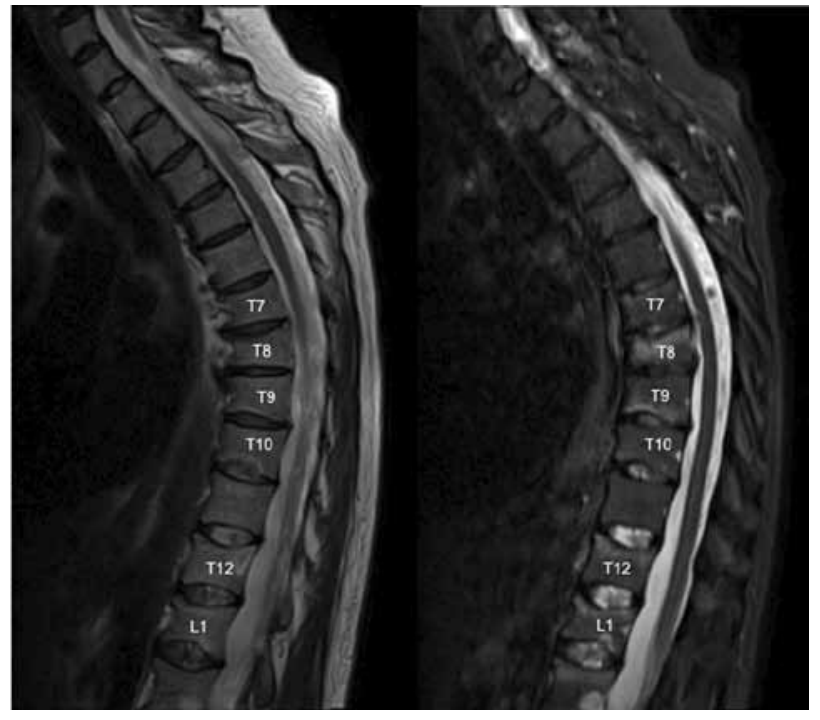

Figure. Sagittal MRI examination of thoracic and upper lumbar spine, before initiation of therapy, revealed vertebral fractures at T7-T10, T12 and L1.

The physical examination was normal without neurological signs, except for the thoracolumbar back pain and an apparent mild thoracic kyphosis. A thorough investigation revealed low vitamin D levels (Table 1) without other abnormalities, indicating that the patient did not have any other cause of secondary osteoporosis. Bone densitometry measured by Lunar Prodigy dual-energy X-ray absorptiometry (DXA) was consistent with bone mineral density lower than that expected for the age (z-score) in the lumbar spine and the hip (Table 2). Thus, the patient was diagnosed as having PLO. Informed consent was obtained from the patient.

Breast-feeding was terminated (two months after delivery) and treatment was started with calcium 500 $\mathrm{mg} /$ day, vitamin $\mathrm{D}_{3} 2.200 \mathrm{IU} /$ day and TRP $20 \mu \mathrm{g} /$ day. Shortly after the initiation of TRP treatment, the back pain gradually decreased and the patient stopped the NSAIDs. After one month of TRP treatment, she was able to get up from bed and after 80 days she returned to work.

At her recent follow-up 13 months after the initiation of the therapy, the patient was almost free of back pain but experienced back stiffness. There was no new clinical vertebral fracture. Her laboratory tests were all normal (Table 1). BMD increased by $24.4 \%$ at the lumbar spine, $9.9 \%$ and $4.6 \%$ at the left and 
Table 1. Serum and urinary laboratory tests of the patient before and after the initiation of teriparatide treatment

\begin{tabular}{|c|c|c|c|c|c|c|}
\hline Value & Normal range values & Before treatment & After 1 mo & After 4 mo & After 7 mo & After 13 mo \\
\hline \multicolumn{7}{|l|}{ Serum } \\
\hline Calcium (mg/dl) & $8.4-10.1$ & 9.9 & 9.5 & 8.7 & 9.7 & 9.6 \\
\hline Phosphate (mg/dl) & $2.7-4.5$ & 4.0 & 4.3 & 4.2 & 4.4 & 4.3 \\
\hline ALP (iu/l) & $35-104$ & 68.0 & & & & 43.0 \\
\hline 25-OH-vit D3 (ng/ml) & $30-150$ & 15.5 & 30.7 & 16.7 & 49.0 & 33.5 \\
\hline PTH (pg/ml) & $15-65$ & 14.0 & 40.0 & 38.0 & 28.0 & 21.0 \\
\hline PINP (ng/ml) & $15-59$ & 40.0 & 149.3 & 81.5 & 50.5 & 40.0 \\
\hline $\mathrm{OC}(\mathrm{ng} / \mathrm{ml})$ & $11-43$ & & & & & 26.9 \\
\hline TSH $(\mu \mathrm{iu} / \mathrm{ml})$ & $0.55-4.78$ & 3.11 & & & & 1.93 \\
\hline \multicolumn{7}{|l|}{ Urinary $24 / \mathrm{h}$} \\
\hline $\mathrm{Cr}(\mathrm{mg} / 24 \mathrm{~h})$ & $800-1,800$ & 983 & 929 & 807 & 1,066 & \\
\hline Calcium (mg/24h) & $100-300$ & 86 & 165 & 158 & 197 & \\
\hline Phosphate (mg/24h) & $350-1,400$ & 689 & & & & \\
\hline
\end{tabular}

ALP: alkaline phosphatase; 25-OH-vit D3: 25 hydroxy vitamin D; PTH: parathyroid hormone; PINP: procollagen type I N-terminal propeptide; OC: osteocalcin; TSH: thyroid-stimulating hormone Cr: creatinine.

Bold text indicates values out of normal range.

Table 2. Bone mineral density (BMD) of the L1-L4 and right and left hip: BMD as absolute values ( $\mathrm{g} / \mathrm{cm} 2), \mathrm{Z}$ score and the changes after one year of teriparatide treatment

\begin{tabular}{lccccc}
\hline & Before treatment & & After 13 mo & Change (\%) \\
\hline L1 & BMD & Z-score & BMD & Z-score & \\
L2 & 0.748 & -3.1 & 0.880 & -1.8 & 17.6 \\
L3 & 0.624 & -4.7 & 0.781 & -3.2 & 25.2 \\
L4 & 0.595 & -4.9 & 0.763 & -3.4 & 28.2 \\
L1-L4 & 0.592 & -4.9 & 0.748 & -3.5 & 26.4 \\
Femur neck (R) & 0.634 & -4.4 & 0.789 & -3.0 & 24.4 \\
Total hip (R) & 0.628 & -2.7 & 0.677 & -2.2 & 7.8 \\
Femur neck (L) & 0.697 & -2.3 & 0.729 & -2.0 & 4.6 \\
Total hip (L) & 0.617 & -2.8 & 0.695 & -2.0 & 12.6 \\
\hline
\end{tabular}

(R): right; (L): left; *The same densitometer was used for all bone mineral density measurements.

the right total hip and $12.6 \%$ and $7.8 \%$ at the left and right femur neck, respectively (Table 2).

\section{DISCUSSION}

Pregnancy and lactation itself can affect bone metabolism. Indeed, during the third trimester of pregnancy considerable amounts of calcium are transported from the maternal skeleton to the foetus resulting in increased maternal bone turnover. It should be noted here that during pregnancy powerful compensatory mechanisms take effect protecting the maternal skeleton consisting of increased calcium absorption and high oestrogen levels. Nevertheless, there is a definite post-partum decrease in maternal BMD by $1.8-3.2 \%$, depending the skeletal site. ${ }^{8}$ Additionally, the even greater calcium losses during lactation (approximately $300 \mathrm{mg}$ calcium/day) further increase the risk of PLO. ${ }^{9,10}$ During the first month of lactation, bone resorption markedly rises 
and subsequently normalizes, while bone formation increases and remains elevated for at least one more month. Interestingly, loss of calcium induced by lactation may not be the only cause of the loss of bone density in lactating women. Lactating women have lower oestrogen levels, higher prolactin levels, higher parathormone-related peptide (PTH-related peptide) levels and lower $1,25(\mathrm{OH})_{2} \mathrm{D}$ levels than non-lactating women. ${ }^{9}{ }^{11}$ During lactation women experience a BMD loss of approximately $3-7 \%$. The reversal of physiological oestrogen fall, increased PTH-related peptide and the maternal calcium loss in breast milk during lactation lead to recovery of bone loss in most of the patients. ${ }^{8,12}$

PLO is a rare disorder of unclear aetiology. However family history, vitamin D deficiency, low body weight, prior low BMD and smoking may be predisposing factors. ${ }^{5-7}$ Premenopausal osteoporosis risk factors such as hyperthyroidism, the use of medications that can adversely affect the skeleton (LMWH, glycocorticoids), etc. should be investigated and the underlying cause must be treated first. ${ }^{13}$

We report the case of a patient suffering from PLO with severe back pain who was not able to get up from bed. She presented with 6 vertebral fractures until the initiation of the TRP treatment. Both spine and hip BMD in our patient was significantly lower than the expected normal values for her age. These findings suggested a disease of great severity.

In this patient, the use of LMWH during the last 2 trimesters of pregnancy was a risk factor..$^{14}$ Although the mechanism is unclear, there are indications that long-term LMWH use (over three months) decreases bone formation and accelerates bone resorption. ${ }^{15}$ However, pregnant women who required prophylactic doses of LMWH or unfractionated heparin and received calcium supplementation during pregnancy did not appear to have a clinically significant decrease in BMD. ${ }^{16}$ The nodular thyroid disease treated with levothyroxine for one year in the past with normal TSH levels ever since and the treatment with contraceptive pills for 21 days only, before artificial fertilisation, could not be considered as risk factors. ${ }^{17,18}$ Moreover, although vitamin D deficiency has been documented in PLO patients, this was not the case with our patient. ${ }^{5}$
In some patients the clinical symptoms of PLO resolve spontaneously, while others have severe, prolonged and disabling back pain. These patients require therapy of PLO. There is no consensus to date on how to treat PLO since, while there are a few reported cases, there are no clinical trials. In a case series, patients with PLO treated with weaning and calcium with or without supplementation of vitamin D presented increase of spine BMD by $6.2 \%$ at $8-18$ months and $9.5 \%$ at 2-4 years and the hip BMD increased by $4.1 \%$ and $4.4 \%$, respectively. ${ }^{3}$

Antiresorptive drugs are considered first-line treatment for osteoporosis and their use has been reported increasingly in PLO ${ }^{19} \mathrm{BP}$ treatment for PLO had much better outcomes than weaning and calcium and vitamin D supplementation. After BP treatment, the spinal BMD improved by $17 \%$ in one year and $23 \%$ in two years, while the hip BMD improved by only $0.7 \%$ in one year and remained unchanged in the second year of treatment. ${ }^{5}$ However, the long-term effects of BPs are not well known. Concern about BP use in premenopausal women is related to the increased calcium requirements during pregnancy and lactation as well as the pharmacokinetic and pharmacodynamic properties of BPs that result in prolonged retention in the skeleton and suppression of bone turnover. In addition, animal data point to a transplacental passage of BP and significant maternal and offspring toxicity, although the doses used in these animal studies were much higher compared to those approved for use in humans. ${ }^{4}$

Strontium ranelate has been used as an alternative therapy. In a case of PLO, the spine BMD increased by $33 \%$ in 21 months and by $40 \%$ in 34 months and the hip BMD increased by $20.5 \%$ and then remained unchanged, respectively. ${ }^{20}$ Although the results of this case were also satisfactory, strontium ranelate is a drug with unclear mechanism of action and which is not approved by the FDA and its benefits and harms need to be established. Furthermore, it has been suggested that it is very difficult to assess strontium-induced changes in bone mineral density. Thus, alternative therapies, such as TRP, must be considered.

The most effective treatment of premenopausal osteoporosis may be with the use of an anabolic agent such as parathyroid hormone (PTH). TRP, the 
human recombinant PTH (1-34), has been assigned to pregnancy category $\mathrm{C}$ by the FDA. It improves BMD and bone strength and has a serum half-life of 1 hour. ${ }^{21}$ Thus, if TRP treatment ceases before a future gestation, there will be no adverse effects on the mother and the foetus. These data, combined with the severity of PLO and the improvement of back pain in postmenopausal women with vertebral fractures after TRP treatment, led us to the decision to manage our patient with TRP. ${ }^{22}$ After 13 months of treatment, BMD was increased by $24.4 \%$ at the lumbar spine, $9.9 \%$ and $4.6 \%$ at the left and the right total hip, and $12.6 \%$ and $7.8 \%$ at the left and right femur neck, respectively, without any further fracture occurrence. To our knowledge, there are three articles reporting the outcomes of TRP treatment in PLO. ${ }^{2,23,24}$ In one patient, after 18 months of therapy, the increase of BMD at the spine and total hip was $36 \%$ and $13.8 \%$, respectively. ${ }^{24}$ In a small case series, three PLO patients improved their BMD by $14.5-25 \%$ at the lumbar spine and $13.4-17.9 \%$ at the total hip. ${ }^{2}$

In summary, women with PLO may suffer from vertebral fragility fracture(s), often multiple, which cause severe and disabling back pain and kyphosis in the third trimester of pregnancy or in early postpartum. Treatment with TRP, which stimulates bone formation, simultaneously with weaning and calcium and vitamin D supplementation, seems to considerably increase BMD, improve severe back pain and quality of life and prevent further occurrence of vertebral fractures, making TRP a helpful tool in restoring bone strength in PLO patients.

\section{Declaration of interest}

The authors do not have any conflict of interest to declare.

\section{Funding}

This research did not receive any specific grant from any funding agency in the public, commercial or not-for-profit sector.

\section{REFERENCES}

1. Nordin BE, Roper A, 1955 Post-pregnancy osteoporosis; a syndrome? Lancet 268: 431-434.

2. Choe EY, Song JE, Park KH, et al, 2011 Effect of teriparatide on pregnancy and lactation-associated osteoporosis with multiple vertebral fractures. J Bone Miner Metab 30: 596-601.

3. Phillips AJ, Ostlere SJ, Smith R, 2000 Pregnancyassociated osteoporosis: does the skeleton recover? Osteoporos Int 11: 449-454.

4. Stathopoulos IP, Liakou CG, Katsalira A, et al, 2011 The use of bisphosphonates in women prior to or during pregnancy and lactation. Hormones (Athens) 10: 280-291.

5. O'Sullivan SM, Grey AB, Singh R, Reid IR, 2006 Bisphosphonates in pregnancy and lactation-associated osteoporosis. Osteoporos Int 17: 1008-1012.

6. Di Gregorio S, Danilowicz K, Rubin Z, Mautalen C, 2000 Osteoporosis with vertebral fractures associated with pregnancy and lactation. Nutrition 16: 1052-1055.

7. Dunne F, Walters B, Marshall T, Heath DA, 1993 Pregnancy associated osteoporosis. Clin Endocrinol (Oxf) 39: 487-490.

8. Moller UK, Vieth Streym S, Mosekilde L, Rejnmark L, 2012 Changes in bone mineral density and body composition during pregnancy and postpartum. A controlled cohort study. Osteoporos Int 23: 1213-1223.

9. Uemura H, Yasui T, Kiyokawa M, et al, 2002 Serum osteoprotegerin/osteoclastogenesis-inhibitory factor during pregnancy and lactation and the relationship with calcium-regulating hormones and bone turnover markers. J Endocrinol 174: 353-359.

10. Kovacs CS, 2011 Calcium and bone metabolism disorders during pregnancy and lactation. Endocrinol Metab Clin North Am 40: 795-826.

11. Sowers MF, Hollis BW, Shapiro B, et al, 1996 Elevated parathyroid hormone-related peptide associated with lactation and bone density loss. JAMA 276: 549-554.

12. Kalkwarf HJ, Specker BL, 2002 Bone mineral changes during pregnancy and lactation. Endocrine 17: 49-53.

13. Bhalla AK, 2010 Management of osteoporosis in a premenopausal woman. Best Pract Res Clin Rheumatol 24: 313-327.

14. Deruelle P, Coulon C, 2007 The use of low-molecularweight heparins in pregnancy--how safe are they? Curr Opin Obstet Gynecol 19: 573-577.

15. Muir JM, Hirsh J, Weitz JI, Andrew M, Young E, Shaughnessy SG, 1997 A histomorphometric comparison of the effects of heparin and low-molecular-weight heparin on cancellous bone in rats. Blood 89: 3236-3242.

16. Casele H, Haney EI, James A, Rosene-Montella K, Carson M, 2006 Bone density changes in women who receive thromboprophylaxis in pregnancy. Am J Obstet Gynecol 195: 1109-1113.

17. Larijani B, Gharibdoost F, Pajouhi M, et al, 2004 Effects of levothyroxine suppressive therapy on bone mineral density in premenopausal women. J Clin Pharm Ther 29: $1-5$.

18. Almstedt Shoepe H, Snow CM, 2005 Oral contraceptive use in young women is associated with lower bone mineral density than that of controls. Osteoporos Int 16: 
1538-1544.

19. Neff MJ, 2004 ACOG releases guidelines for clinical management of osteoporosis. Am Fam Physician 2004 69: 1558-1560.

20. Tanriover MD, Oz SG, Sozen T, Kilicarslan A, Guven GS, 2009 Pregnancy- and lactation-associated osteoporosis with severe vertebral deformities: can strontium ranelate be a new alternative for the treatment? Spine J 9: e20-24.

21. Quattrocchi E, Kourlas H, 2004 Teriparatide: a review. Clin Ther 26: 841-854.

22. Genant HK, Halse J, Briney WG, Xie L, Glass EV, Krege
$\mathrm{JH}, 2005$ The effects of teriparatide on the incidence of back pain in postmenopausal women with osteoporosis. Curr Med Res Opin 21: 1027-1034.

23. Stumpf UC, Kurth AA, Windolf J, Fassbender WJ, 2007 Pregnancy-associated osteoporosis: an underestimated and underdiagnosed severe disease. A review of two cases in short- and long-term follow-up. Adv Med Sci 52: 94-97.

24. Hellmeyer L, Boekhoff J, Hadji P, 2010 Treatment with teriparatide in a patient with pregnancy-associated osteoporosis. Gynecol Endocrinol 26: 725-728. 